\title{
Ultrasound-guided continuous costoclavicular brachial plexus block - a new approach for pain management in paediatrics physiotherapy
}

Dias M.B. ${ }^{1}$, Artilheiro V. ${ }^{2}$, Regufe R. ${ }^{3}$, Costa G. ${ }^{4}$, Cenicante T. ${ }^{4}$, Peixer I. ${ }^{4}$, Ferreira J.L. ${ }^{4}$

${ }_{1}^{1}$ Hospital da Luz Lisboa, Anaesthesiology Department, Lisboa, Portugal

${ }^{2}$ Centro Hospitalar de Lisboa Ocidental, Anaesthesiology Department, Lisboa, Portugal

${ }^{3}$ Hospital de Setúbal, Anaesthesiology Department, Lisboa, Portugal

${ }^{4}$ Centro Hospitalar Universitário de Lisboa Central, Anaesthesiology Department, Lisboa, Portugal

\section{Background}

- Since its first description in $2015^{[1]}$, ultrasound-guided costoclavicular brachial plexus block has been increasingly used for anaesthesia and analgesia of the upper limb.

- To our knowledge, there are no reports of its use for pain management during physical therapy.

- We describe the first continuous ultrasound-guided costoclavicular brachial plexus block for physical rehabilitation after a traumatic elbow fracture in a child.

\section{Clinical case}

- 12-year-old, 30kg, healthy child, ASA 1

Traumatic ellow fracture-dislocation with intra-articular bone fragment

Conservative treatment
Significant posttraumatic painful stiffness
No collaboration in rehabilitation process $\checkmark$ Effective pain control (VAS $8 \Rightarrow 3$ )

$\checkmark$ Re-engagement in physiotherapy

$\checkmark$ Progressive gain in elbow motion

Ultrasound-guided continuous costoclavicular brachial plexus block

- $5 \mathrm{~mL}$ ropivacaine $0.2 \%$ bolus 20 min prior each rehabilitation session (total of 7 treatments)

Catheter removal after 15 days

(filter changed weekly)

\section{Discussion}

- Continuous costoclavicular peripheral nerve block was a successful alternative to pain management in this post-traumatic rehabilitation setting.

- Continuous regional analgesia through costoclavicular approach of brachial plexus may be a valuable option not only for pain control but also for improving functional prognosis during rehabilitation in the paediatric population.

\section{References}

[1] Karmakar MK, Sala-Blanch X, Songthamwat B, Tsui BC. Benefits of the costoclavicular space for ultrasound-guided infraclavicular brachial plexus block: description of a costoclavicular approach. Reg Anesth Pain Med 2015;40:287-288. 